\title{
Kapitel V
}

\section{Der Nutzen von Befriedigung: \\ Ein Zirkel in der Entscheidungstheorie ${ }^{1}$}

\author{
Anna Kusser ind Wolfgang Spohn
}

1. Eine kausale Voraussetzung der Entscheidungstheorie

2. Eine Verletzung dieser Voraussctzung

3. W'ie man dem Zirkel nicht entgeht

4. Wiie man dem Zirkel gerecht werden kann
Erschienen in: Praktische Rationalität : Grundlagenprobleme und ethische Anwendungen des rational choice-Paradigmas / Nida-Rümelin, Julian (Hrsg.). - Berlin : De Gruyter, 1994. (Perspektiven der analytischen Philosophie ; 2). - S. 169-196. - ISBN 978-3-11-087155-5

https://dx.doi.org/10.1515/9783110871555.169

Niemand wird bezweifeln, daß wir intrinsische Wünsche haben. Dcnn sicherlich haben wir Wünsche, und viele von ihnen sind abgeleitet. Demnach müssen - wie bei einer deduktiven Theorie, deren beweisbare Aussagen in Axiome und Theoreme zerfallen - einige unserer Wünsche grundlegend sein.

Weit weniger klar ist dagegen, worauf sich unserc intrinsischen Wünsche bezichen oder beziehen solltcn. Unbestreitbar dürftc immerhin sein, daß neben anderem Glück, Freudc, Schmerzfreiheit, gewisse Empfindungsqualitäten, Gefühle und Stimmungen, eben allgemein die eigenen psychischen Befindlichkeiten, Gcgenstand intrinsischer Wünsche sind; die Wünsche danach scheinen einer weiteren Begründung wedcr fähig noch bedürftig.

Wünsche sind mchr oder weniger stark, und sie bestimmen, zusammen mit mehr oder weniger festen Überzeugungen, rationales Handeln. Den unterschiedlich starken Wünschen und Überzeugungen entsprechen im

1 Diese Arbeit ist einc Ubbersetzung und leichte Uberarbcitung unseres Aufsat\%cs ,I The Utility of Pleasure is a Pain for Decision Theory", Journal of Pbilosopby 89 (1992), S. 10-29; sie erscheinc hier mit freundlicher Genehmigung des Jormal of Philosopby. W'ir sind sehr Jankbar für die Gelegenheiten zur Diskussion dicses Aufsatzcs, die uns an der Freien Universität Berlin, an der Universität von Tilburg, der University of California at Irvine und der Universität cles Saarlandes geboten wurden. Insbesondere danken wir Jack Birner, David Gauthier, Bert Hamminga, Dan Hausman, Isaac Levi, Gregory Kavka, Karel Lambert, Alan Nelson, Rainer Trapp und Ernst Tugendhat für hilfreiche Kommentare. Die Arbeit wurde tcilweise durch die Deutsche Forschungsgemeinschaft unter dem Vorhaben Nr. Sp 279!2-1 unterstützt. 
formalen Rahmen der Entscheidungstheorie die subjektiven Nutzenwerte und Wahrscheinlichkeiten. Auch die Unterscheidung zwischen intrinsischen und extrinsischen Wünschen findet in der Entscheidungstheorie ihren Niederschlag, da jedes Modell eine - jedenfalls relativ zum jeweiligen Modell - grundlegende Nutzenfunktion enthält sowie eine Funktion des erwarteten Nutzens, die sich aus der grundlegenden Nutzenfunktion und der subjektiven Wahrscheinlichkeitsfunktion herleitet. ${ }^{2}$ In der Tat kann die Entscheidungstheorie für sich beanspruchen, die soweit insgesamt beste formale Explikation der intuitiven, von Wünschen und Überzcugungen ausgehenden praktischen Überlegung zu liefern. Mithin ist davon auszugehen, daß die intrinsisch gewünschten psychischen Zustände einer Person im Definitionsbereich ihrer Nutzenfunktion liegen. Dann aber, so behaupten wir, versagt die Entscheidungstheorie. Genauer gesagt, wollen wir zwei Thesen verteidigen:

(A) Wenn Entscheidungssituationen eine gewisse kausale Struktur aufweisen, kommt die Fntscheidungstheorie in Schwierigkeiten, da sich in ihnen kein erwarteter Nutzen bestimmen läßt.

(B) Entscheidungssituationen dieser kausalen Struktur liegen tatsächlich häufig vor, nämlich in der Regel dann, wenn die Nutzenfunktion für intrinsisch evaluierte psychische Zustände definiert ist.

Der Abschnitt 1 erläuter die These (A); im Abschnitt 2 wird die These (B) verteidigt. Dem Verdacht, daß die aufgewiesene Unzulänglichkeit der Entscheidungstheorie auf irgendwelchen Mißverständnissen beruht, versuchen wir im Abschnitt 3 zu begegnen. Obwohl wir für unser Problem keine Lösung haben, und uns nicht einmal ganz klar ist, was man eine Lösung nennen könnte, sollen im letzten Abschnitt 4 einige konstruktive Folgerungen gezogen werden.

Da das Problem nicht Produkt einer bestimmten Formalisierung der Entscheidungstheorie ist, werden wir die praktische Überlegung und die Entscheidungstheorie nur in informeller oder halbformaler Weise behan-

2 In der klassischen Theoric von Savage (1954) ist die grundlegende Nutzenfunktion für Konsequenzen erklärs, und Handlungen erhalten erwartete Nuezenwerte. In der Standardtheorie der Enuscheidungsbāume (vgl. etwa Raiffa, 1968, Kap. 2) ordnet die grundlegende Nuzzenfunktion entweder jedem Knoten oder jedem Ast eines Entscheidungsbaumes einen Wert zu. In einem von den Endpunkten zum Ursprung des Baumes laufenden rekursiven Venfahres wird dann jedem Knoten im Hinblick auf dem bei ihm beginnenden Teilbaum ein erwarteter Nutzenwert zugewiesen. In der wieder etwas anderen Theorie von Jeffrey (1965) werden schließlich vollständigen konsistenten Geschichten oder möglichen Welten grundlegende nicht-probabilistische Nutzenwerte zugeordnet, und Propositionen, die aus mehr als einer möglichen Welt bestehen, erhalten erwante Nutzenwerte. 
deln. Doch lassen sich unsere Ausführungen formal leicht mit Hilfe von Entscheidungsbäumen oder Fintscheidungsflußdiagrammen nachvollziehen, wie sie in Raiffa (1968) exemplarisch dargestellt sind. Seine Theorie kann daher nötigenfalls als formale Leitlinie dienen; und auf sie beziehen sich unsere Aussagen zur formalen Fntscheidungstheorie, auch wenn unsere Intentionen natürlich allgemeiner sind.

Wie sich zeigen wird, ist das hier behandelte Problem nichts anderes als eine Variante von Joseph Butlers Kritik am Hedonismus. ${ }^{3}$ So gesehen laufen unsere Ausführungen darauf hinaus, daß die modernen Theorien der praktischen Überlegung Butlers Kritik nicht ernsthaft aufgenommen haben.

\section{Fine kausale Voraussetzung der Fintscheidungstheorie}

Unserer Thesc (A) zufolge enthält die Entscheidungstheorie eine verstcckte Voraussetzung über die kausale Struktur von Entschcidungssituationen. ${ }^{4}$ Betrachten wir, um diese Voraussetzung aufzudecken, wie wir in Entscheidungssituationen praktisch überlegen. Wir tun dies, um eine Handlung zu finden, die unsere Zicle optimal befördert. Dazu sind einerseits die Ziele, d. h. die intrinsisch gewünschten Sachverhalte, und andererseits die möglichen Handlungen aufzulisten. Jedem Ziel kommt ein Nutzenwert zu, und jedem Ziel und jedcr Handlung eine bedingte Wahrscheinlichkeit, nämlich die Wahrscheinlichkeit dafür, daß sich das Ziel realisiert unter der Bedingung, daß die Handlung ausgeführt wurde. Daraus resultiert für jede Handlung ein erwarteter Nutzen. Und so läßt sich schließlich eine Handlung mit maximalem erwarteten Nutzen bestimmen, die also unsere Ziele optimal befördert.

Der erwartete Nutzen von Handlungen scheint hier in einem cinzigen Schritt berechnet zu werden. In der Regel ist das aber nicht der Fall; wir gehen normalerweise in unseren Übcrlegungen von einem sehr viel reicheren kausalen Bild der Welt aus, welches die vielfältigen Wege berücksichtigt, auf denen unsere Handlungen unsere Ziele beeinflussen. Dies

${ }^{3}$ Vgl. Butler (1729), Preface, of 29-31 und Sermon XI, SS 1-10. Vgl. auch H. Sidgwick (1874), der sich mit dieser Kritik im Buch l, Kap. IV und Buch II, Kap. III, $\int 5$ auseinandersetzt.

4 Es gibt zumindest noch einc andere Vorausseczung dieser Art. Aufgrund dessen, was als ,Newcombs Problem' in die Literatur eingegangen ist, ist die Entscheidungstheorie in zwei Versionen zerfallen, die sich nur unter speziellen Voraussctzungen wieder decken; vgl. die Aufsätze in CampbelliSowden (1985). 
schlägt sich in einer vollständigeren Beschreibung der praktischen Überlegung nieder:

Danach ist es ihr Zweck, wie zuvor den Gesamtnutzen jeder möglichen Handlung und, wie gleich zu sehen sein wird, zudem jedes in Betracht gezogenen Sachverhalts zu bestimmen. Ganz natürlich ist dabei die Annahme, daB der Gesamtnutzen $U^{\prime}(A)$ eines Sachverhalts $A$ einfach die Summe seines intrinsischen Nutzens $U^{\prime}(A)$ und seines extrinsischen Nutzens $U^{\prime}(A)$ ist; ${ }^{5}$ diese Annahme kann freilich nicht als Definition gelten, da sie, wie sich noch herausstellen wird, falsch ist. Man beachte, daß ein Sachverhalt sowohl intrinsischen wie auch extrinsischen Nutzen haben kann: Für manche Leute ist Geld nicht bloß ein Mittel zu einem angenehmen Leben, sondern besitzt schon an und für sich einen Zauber; Autofahren bringt einen schnell, aber unter Lebensgefahr dahin, wo man hin will, und daneben macht es, gelegentlich, als solches Spaß.

Den Ausgangspunkt der praktischen Überlegung bilden demnach die intrinsischen Nutzenwerte der in Betracht gezogenen Sachverhalte (von denen viele gleich Null sein werden). Dann werden auf rekursive Weise der extrinsische und der Gesamtnutzen jedes Sachverhalts bestimmt. Der extrinsische Nutzen eines Sachverhalts $A$ ist ein erwarteter Nutzen; er ist das gewichtete Mittel der Gesamtnutzen der von $A$ direkt kausal beeinflußten Sachverhalte, wobei die Gewichte in den durch $A$ bedingten subjektiven Wahrscheinlichkeiten dieser Sachverhalte bestehen. Damit kann die Handelnde ihr kausales Bild von seinen Endpunkten, d. h. von der Zukunft her aufrollen und so Schritt für Schritt den extrinsischen Nutzen und mit Hilfe der genannten Annahme auch den Gesamtnutzen eines jeden Sachverhalts ermitteln. Zuletzt erreicht sie die Anfangspunkte ihres kausalen Bildes, d. h. die ihr offenstehenden Handlungen und kann so schließlich eine Handlung mit maximalem Gesamtnutzen bestimmen.

W'ie auch immer dieses Bild der praktischen Überlegung im einzelnen formalisiert wird, ${ }^{6}$ zentral ist die in ihm enthaltene Konzeption von Wün-

5 Die Nuczenfunktion bezieht sich natürlich immer auf eine bestimmte Person zu einer bestimmten Zeit, auch wenn das nicht eigens vermerkt ist.

- Diese Skize stimmt mir den üblichen Versionen der Entscheidungstheorie gut überein. Das einschritrige Verfahren enspricht der anfanglichen Darlegung der Entscheidungstheorie ron Savage (1954) im Kap. 2, wo Entscheidungssituationen mit Weltzuständen, Handlungen und Konsequenzen erfaßt werden. Das mehrschrittige Verfahren wird mit Milfe von Enscheidungsbāumen formalisiert - vgl. Raiff (1968), Kap. 2; es ist übrigens auch schon in der Theorie der sogenannten kleinen Welten von Savage (1954), Abschn. 5.5, enthalten - vgl. dazu auch Spohn (1978), Abschn. 2.3 und 3.6. Unsere Ausführungen sind auf diese Standardformalisienung abgestimmt. Schließlich tritt der kausale Charakter der praktischen Überlegung in allen Versionen der kausalen Entscheidungstheorie klar zueage, die ja als eine Ausarbeitung von Savages Theorie intendier sind; vgl. ecwa Skyrms (1984), Kap. 4. 
schen oder Nutzenwerten sowie der zwischen ihnen bestehenden Ableitungsbeziehung: Es gibt nur intrinsischen und extrinsischen Nutzen und Summen davon; dic intrinsischen Nutzenwerte sind nicht abgeleitet, die extrinsischen sind abgeleitet, und die Ableitung schrcitet von den vermuteten kausalen Folgen und ihren Wahrscheinlichkeiten und Gesamtnutzenwcrten zu den vermuteten Ursachen und deren extrinsischen Nutzenwerten fort.

$\mathrm{Da}$ wir von $A$ bleitung reden, mag den Eindruck erwecken, als faßten wir die Entschcidungstheorie als eine syntaktische Theorie der rationalen Berechnung von Nutzenfunktionen (und Wahrscheinlichkeiten) auf. Dcm ist nicht so. Nach unscrem Verständnis formuliert die F.ntscheidungstheoric vielmehr Koexistenzgesetze für Nutzen- und Wahrscheinlichkeitsfunktionen im Rahmen rationaler Überzeugungs- und Wunschkomplexe. Der eher bildliche Ausdruck, daß ein Teil eines solchen Komplexes sich aus anderen 'Teilen herleitet, soll hier also durchweg nichts anderes bedeuten, als daß dieser Teil gemäß den entscheidungstheoretischen Gesetzen durch die anderen eindeutig bestimmt ist.

Wenn nun die praktische Čberlegung wie cben beschrieben funktionieren soll, so müssen einige Bedingungen kausaler Wohlgeordnetheit erfüllt sein. Eine natürliche Annahme besteht darin, daß der in die praktische Ubberlegung eingehende Komplex von Wünschen und Übcrzeugungen hinreichende Ursache des tatsächlichen Verhaltens ist. Diese Annahme gilt per definitionem für alle rational Handelnden.' Sie kann insofern als Voraussetzung jeder praktischen Überlegung gelten, als die Überlegung umso witzloser ist, je weniger sie aufs Handeln durchschlägt. Aus der Verletzung dieser Voraussetzung resultieren Anomalien, die unter dem Stichwort ,Willensschwäche' viel diskutiert werden. ${ }^{\text {' }}$

Fine anderc Voraussetzung - und um sie geht es hier - lautet, daß das Frgebnis eines jeden Überlegungsschrittes nicht schon bei einem früheren Überlegungsschritt vorausgcsetzt werden darf; andernfalls gcriete die gesamte Überlegung offenbar in einen Zirkel. Das Ergebnis eines Überlegungsschritts sind extrinsische Nutzen- und Gesamtnutzenwerte. Wie ist es angesichts der Azyklizität der Kausalbeziehung möglich, daß dicse bei einem früheren Überlegungsschritt benötigt werden? Das ist

'Die Kausalisten unter den Handlungstheoretikern sind jedenfalls der Auffassung, daß praktische Gründe Handlungen verursachen können und dies im Falle rationalen Handelns auch tun. Vigl. etwa die ersten drei Aufsätze in Davidson (1980) oder Churchland (1970).

* Vgl. u. a. den zweiten Aufsatz in Davidson (1980), Pears (1986) und Kusser (1989), Abschn. 3.1 und 4.2. 
offenbar gerade dann der Fall, wenn die Tatsache, daß ein Sachverhalt für ein Subjekt einen bestimmten extrinsischen oder Gesamtnutzen hat, für diejenigen W'irkungen dieses Sachverhalts kausal relevant ist, aus deren Nutzen sich sein extrinsischer und sein Gesamtnutzen herleitet.

Betrachten wir zunächst ein einfaches abstraktes Beispiel dieser, wie es scheint, absonderlichen Konstellation. Nehmen wir dazu an, daß der Gesamtnutzen von $B$ und $\sim B^{9}$ für ein Subjekt bereits bestimmt und $A$ der einzige Sachverhalt sei, der für $B$ und $\sim B$ kausal relevant ist. Die Ableitung des extrinsischen und des Gesamtnutzens von $A$ liegt dann auf der Hand:$$
U^{\prime}(A)=U^{\prime}(B) P(B \mid A)+U^{\prime}(\sim B) P(\sim B \mid A),
$$$$
U^{\prime}(A)=U^{i}(\Lambda)+U^{\prime}(A) \text {. }
$$

Nehmen wir nun weiter an, daß $B$ aus der Sicht der Handelnden unter anderem auch davon kausal abhängt, welchen Gesamtnutzen $A$ für sie hat. In diesem Fall wäre die Wahrscheinlichkeit $P(B \mid A)$ in (1) nicht zu verwenden; vielmehr müßte man in der Herleitung für alle möglichen Werte $x$ von $U^{\prime}(A)$ die Wahrscheinlichkeiten $P\left(B \mid A \& U^{\prime}(A)=x\right)$ heranziehen. ${ }^{10}$ Dann wird jedoch die entscheidungstheoretische Herleitung offenkundig zirkulär; denn um den Wert $U^{\top}(A)$ entsprechend dem modifizierten (1) herzuleiten, muß man bereits den Wert von $U^{\prime}(A)$ kennen, der aber, (2) zufolge, davon abhängt, welchen Wert $U^{\prime}(A)$ annimmt. Demnach kann also die cntscheidungstheoretische Nutzenherleitung nur funktionieren, wenn eine solche fatale kausale Abhängigkeit nicht besteht.

Wir wollen natürlich behaupten, daß diese Abhängigkeit weder unmöglich noch absonderlich, sondern im Gegenteil ganz normal ist. Doch sind zunächst noch einige Bemerkungen auf abstrakter F.bene am Platze:

Erstens geht unser Argument von der Annahme aus, daß kausale Abhängigkeiten sich in der Sicht der Handelnden in stochastischen Abhängigkeiten gemäß ihren subjektiven Wahrscheinlichkeiten spiegeln. Wir halten diese Annahme für korrekt, doch ist hier nicht der Ort, um für diesen schwierigen Punkt zu argumentieren. ${ }^{11}$

Zweitens beruht das Argument auf der Annahme, daß die Wahrscheinlichkeit $P\left(B \mid A \& U^{\prime}(A)=x\right)$ verwendet werden muß, weil $P(B \mid A)$ nicht zur Verfügung steht. Das scheint falsch zu sein. Warum kann man

Die Tilde steht für die Negation von Sachverhalten.

10 $" L^{\prime \prime}(A)=x^{\text {" }}$ steht für den Sachverhalt, $\operatorname{da}$ der Gesamtnutzen von $A x$ ist.

1 Zu unserer Ansicht über den 7.usammenhang zwischen Kausalität und Wahrscheinlichkeit vgl. Spohn (1978), Abschn. $3.2+3$ und 5.1, sowie (1980) und (1990). 
nicht $P(B \mid A)$ durch die Formel von der totalen Wahrscheinlichkeit berechnen, indem man die Summe (oder das Integral) aller Produkte $P(B \mid$ $\left.A \& U^{\prime}(A)=x\right) \cdot P\left(U^{\prime}(A)=x \mid A\right)$ bildet? Das würde allerdings voraussctzen, daß die Handelnde für den eigenen Gesamtnutzen von $A$ einc Wahrscheinlichkcitsverteilung hat (darauf läuft $P\left(U^{\prime}(A)=x \mid \Lambda\right.$ ) ja hinaus); und das mutet seltsam an. Wichtiger ist jedoch, daß diesc Annahme sich sclbst aufhebt; sie würde erlauben, zucrst $P(B \mid \Lambda)$ und dann, mit Hilfe von (1) und (2), $U^{\prime}(\Lambda)$ zu berechnen und so $U^{\prime}(\Lambda)$ mit Sicherheit zu ermitteln. Natürlich spiegelt sich in diescr Selbstaufhebung nichts andcres als die Zirkularität der ins $\Lambda$ uge gefaßten Situation.

Diese Sachlage tritt nur dann nicht auf, wenn man sich von vornherein cines selbst-vcrifizierenden Wertcs von $U^{\prime}(A)$ sicher ist, eines Wcrtes also, der, wenn man in den Berechnungen von ihm ausgeht, hinterher gerade wicder herauskommt. Das ist die I.ösung, die wir im $\Lambda$ bschnitt 4 vorschlagen und erläutern werden. Man beachte jedoch, daß dann von einer Ableitung nicht mehr die Rede sein kann, da es nicht nur einen sich selbst verifizierenden Wert von $U^{\prime}(A)$ zu geben braucht. Und selbst wenn es genau einen solchen Wert gäbc, so ist das Verfahren zur Bestimmung des W'ertes von $U^{\prime}(A)$ doch ein ganz anderes als in dem Fall, wo die fatalc kausale Abhängigkeit nicht besteht. Wie drastisch diese Lösung das üblichc entscheidungstheoretische Bild verändert, läßt sich in vollen Umfang erst crmessen, wenn klar geworden ist, wie diese zirkuläre Situation eintreten kann (s. Abschnitt 2) und wieso man im Rahmen des üblichen Bildes diescn Zirkel nicht los werden kann (s. Abschnitt 3).

Drittens enthalten (1) und (2) dic implizite Annahme, daß der intrinsische Nutzen von $A$ von all den intrinsischen Nutzen unabhängig ist, die in den extrinsischen Nutzen von $A$ cingehen. Diese Annahme ist jedoch nicht wesentlich. Will man Nutzenabhängigkeiten zulassen, so muß man für den oben erläutcrten Überlegungsschritt den Gesamtnutzen von $A$ \& $B$ und den von $A \& \sim B$ als bereits gegeben ansetzen; der Gesamtnutzen dicscr Sachverhalte ist ja entweder identisch mit ihren intrinsischen Nutzen oder in den vorherigen Überlegungsschritten bereits ermittelt worden. Auf dieser Grundlagc erhält man dann den Gesamtnutzen von $A$ allein entsprechend der Formel:

$$
U^{\prime}(A)=U^{\prime}(A \& B) P(B \mid A)+U^{\prime}(A \& \sim B) P(\sim B \mid A) .
$$

Nehmen wir abcr wiederum an, $B$ sei von dem Gesamtnutzen, den die Handelnde $A$ zuschreibt, kausal abhängig. Dann kommt man mit (1') aus gcnau den gleichen Gründen in genau die gleichen Schwierigkeiten wie mit (1) und (2). Man verliert also nichts und gewinnt aber an Durchsich- 
tigkeit, wenn man die genannte Nutzenunabhängigkeit unterstellt; die Schwierigkeit bei der Ableitung von extrinsischen und Gesamtnutzenwerten wurzelt eben in den Wahrscheinlichkeiten und nicht in möglichen Nutzenabhängigkeiten.

Schließlich sollten wir die kausalc Voraussetzung noch etwas sorgfältiger spezifizieren, die für die Zirkelfrcihcit von (1) und (2) erforderlich ist. Denn vorderhand scheint es, daß die fatale Kausalstruktur überhaupt nicht überraschend ist, da ja die Nutzenfunktion der Handelnden immer für ihr Handeln selbst ${ }^{12}$ und damit auch für alle weiteren Wirkungen ihres Handelns kausal rclevant ist. Und dieser Umstand allein nötigt uns in unserem schematischen Beispiel noch nicht dazu, in der praktischen Überlegung statt der Wahrscheinlichkeit $P(B \mid A)$ die Wahrscheinlichkeit $P(B \mid A$ \& $\left.U^{\prime}(A)=x\right)$ zu verwenden; denn wenn der kausale Finfluß des tatsächlichen Wertes von $U^{\prime}(A)$ auf $B$ von $A$ abgeschirmt wird, ${ }^{13}$ dann ist diese Wahrscheinlichkeit für jeden Wert von $x$ die gleiche und somit auch gleich $P(B \mid A)$. Daraus folgt, daB die praktische Überlegung nur dann in einen Zirkel führt, wenn der Einfluß des Wertes von $U^{t}(A)$ auf $B$ nicht durch $A$ abgeschirmt ist.

Die von der Fntscheidungstheorie vorausgesetzte Kausalannahme lautet demnach: Falls die extrinsischen oder Gesamtnutzenwerte der Sachverhalte $A_{1}, \ldots, A_{m}$ für diejenigen Sachverhalte $B_{1}, \ldots, B_{n}$ kausal relevant sind, aus deren Nutzen sich der extrinsische Nutzen von $A_{\imath}, \ldots, A_{m}$ herleitet, so wird dieser Kausaleinflu $\beta$ von $A_{1}, \ldots, A_{m}$ selbst abgeschirmt. Wann immer diese Voraussetzung verletzt ist, führt die entscheidungstheoretische $\mathrm{Ab}$ leitung des erwarteten und des Gesamtnutzens in einen fatalen Zirkel.

Nunmehr ist auch leicht einzusehen, wieso diese Voraussetzung in der Regel erfüllt ist und warum sie bislang übersehen wurde. 7,war haben unsere Wünsche und Überzeugungen einen erheblichen Einfluß auf unsere Umgebung, doch ist dieser Einfluß ausschließlich durch unsere Handlungen vermittelt und daher auch von ihnen abgeschirmt. ${ }^{14}$ Die Ableitung des erwarteten und des Gesamtnutzens funktioniert daher reibungslos,

12 Dies wird von der obengenannten Annahme über die Handlungsrelevanz der praktischen Überlegung sichergestellt.

13 Dies heißt, daß für alle $\times$ gilt, $\mathrm{da} \beta B$ von $\operatorname{dem}$ Sachverhalt $U^{\prime}(A)=x$ sowohl unter der Bedingung $A$ wic unter der Bedingung $\sim A$ probabilistisch unabhängig ist.

14 Das ist natürlich eine Ubertreibung; unsere Überzeugungen und Wünsche kommen nicht nur in intentionalem Handeln, sondern auch in unabsich dichen Verhaltensweisen zum Ausdruck (was unserer Transparenz guttut). Doch solange wir uns bloß als Handelnde betrachten, wie es in der Entscheidungstheorie und auch im gegenwärtigen Zusammenhang geschieht, können wir an dieser Übertreibung festhalten. 
solange sich die Sachverhalte, von deren Nutzen die Ableitung ihren Ausgang nimmt, nur auf die äußere Welt beziehen; und das ist in den meisten Anwendungen der Entscheidungstheorie sicherlich der Fall.

\section{Eine Verletzung dieser Voraussetzung}

Nach unserer These (B) ist jene merkwürdige kausale Konstellation, die dic praktische Überlegung durcheinanderbringt, durchaus häufig realisiert. Der let\%te Absatz hat schon den entscheidenden Hinweis dafür gegeben, wo nach ihr zu suchen ist; wir müssen unsere eigenen inneren Zustände, ihre Gewünschtheit und ihre kausale Bedingtheit betrachten.

Zunächst gibt es sicherlich viele innere Zustände, die für uns extrinsischen Nutzen haben; so haben beispielswcise Überzeugungen häufig nur instrumentellen Wert, d. h. sic diencn dazu, besser informierte Entscheidungen zu treffen. Doch gibt es darüber hinaus eine Vielzahl von Empfindungen, Gefühlen, Stimmungen, psychischen Grundbefindlichkeiten und anderen inneren Zuständen, die für uns einen erheblichen intrinsischen Nutzen haben, der nicht aus anderen Wünschen hergeleitet ist. ${ }^{15} \mathrm{Wir}$ klassifizieren diese Zustände nach den Situationen, in denen sie auftreten, nach den Formen ihres Ausdrucks, aufgrund ihrer sehr reichen inneren Phänomenologie und schließlich auch aufgrund ihrer Frwünschtheit (unangenehme Gefühle z. B. sind als solche stets unerwünscht). Intrinsisch evaluierte innere Zustände sollen im weiteren satisfaktive $Z$ ustände heißen.

Wie sind satisfaktive Zustände verursacht? Das ist häufig undurchsichtig, wie wir alle nur zu gut wissen. Einige allgemeine Zusammenhänge lassen sich jedoch festhalten. Einesteils stehen unsere satisfaktiven $\mathrm{Zu}$ stände unter dem Dauereinfluß unserer L'mgebung; andernteils hängt dieser Einfluß von der inneren Verfassung ab, auf die er trifft. So genießt man in der Regel eine Einladung bei Freunden, doch ist der Genuß umso ungetrübter, je weniger man gerade im Streß ist. Die Frage ist, welches die kausal relevanten Teile dicser inneren Verfassung sind. Und die entscheidende Antwort ist im gegenwärtigen Zusammenhang, daß dazu jedenfalls gehört, wie sehr jemand einen äußeren Sachverhalt wünscht. Der innere Zustand, in den eine Person gerät, ist also sowohl Produkt der äußeren Situation, die sie crlebt, als auch ihres vorhergehenden inneren

is Damit soll nicht ausgeschlossen sein, daß diese Zustände auch extrinsischen Nutzen haben; aber im folgenden spielt diese Möglichkeit keine Rolle. 
Zustandes; und die die äußere Situation betreffenden Wünsche sind oft relevanter Teil dieses vorhergehenden inneren Zustands.

Es ist nicht schwer, hierfür Beispiele zu finden. Zwei Männcr schauen sich im Fernsehen das Lokalderby an. Die Kickers haben gerade ein Tor geschossen. Der eine ist begeistert, der andere gcreizt. Woher kommt dieser Unterschicd? Der eine ist eben Kickers-Fan, der andere VfB-Anhänger; für den einen hat das 'or positiven Nutzen, für den andercn negativen, da jeder möchte, da/3 seine Mannschaft gcwinnt.

Ein anderes Beispiel: Vor zwei Jahren war eine Frau schwanger, und sic war ganz unglücklich, nun ist sie wieder schwanger und freut sich sehr. Warum? Seinerzeit wollte sie kein Kind, jetz.t hat sie sich ein Kind gewünscht. Das ist eine kausale Erklärung. Zunächst war da der Wunsch nach einem Kind, dann wurde sie schwanger, und nun ist sie glücklich; hätte sie diesen Wunsch, wie vor zwei Jahren, nicht gehabt, hätte die Schwangerschaft nicht diesc Freude ausgelöst.

Vorausgehender Nutzen und nachfolgende Befriedigung brauchen jedoch nicht parallel zu laufen. Alle Arten von Zusammonhängen sind möglich. Es kann sein, daß ich ein Ziel so wichtig nehme, daß ich es ganz verbissen verfolge und so am Ende die Früchte meiner $\Lambda$ nstrengungen nicht mehr genießen kann. Der verzweifelte Wunsch nach etwas, was man entbehrt, kann sich in zwei Richtungen auswirken: Man kann nicht genug davon bekommen und ist permanent frusticrt, oder man freut sich demütig schon an kleinen Mengen. Ja, es gibt sogar Dinge oder Situationen, die nur dann befricdigen, wenn man sie nicht herbeizuführen versucht oder nicht einmal wünscht. ${ }^{16}$

All diese Beispiele weisen die im vorhergehenden Abschnitt als problematisch erwiesenc Kausalstruktur auf:
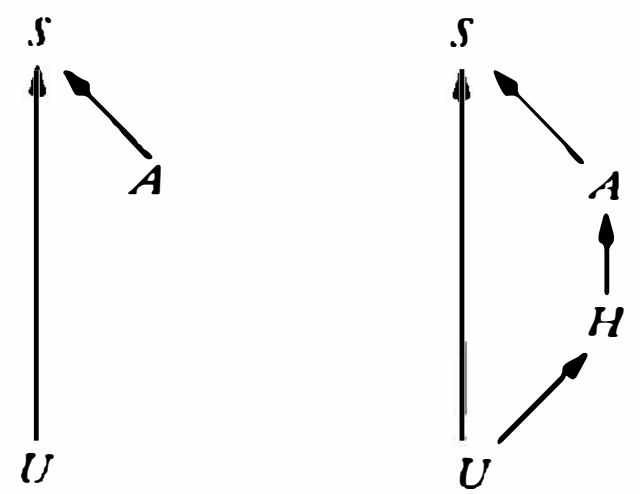

S: ein satisfaktiver Lustand der Person $X$

$A$ : ein äußerer Sachverhalt

$H$ : eine Handlung von $X$

$U$ : $X$ s Haben einer Gesamtnutzenfunktion, in deren Definitionsbereich sich $S, A$ und $H$ befinden

$\rightarrow$ heißt: ist kausal relevant für

Innenwelt Außenwelt Innenwelt Außenwelt von $X$ von $X \quad$ von $X$ von $X$

Abb. A

Abb. B

16 Vgl. Filster (1983), der im Abschn. Il.2 paradoxe Situationen dieser Art diskutier. 
Die Abbildung A zeigt das Grundgerüst diescr Struktur; es ist im Fußballbeispiel realisiert, wobei $A$ die Tatsache ist, da $\beta$ die Kickers gerade ein Tor geschossen haben und $S$ für die Begeisterung des cinen bzw. für die Gereiztheit des anderen steht. Eine Kausalkettc läuft von der äußeren Tatsachc $A$ (via Fernsehen usw.) zum innercn 'Zustand S'. Einc zweite Kausalkette läuft von der Nutzenfunktion $U$ zu $S$, ohne die innere Welt der Person $X$ zu verlassen. Dicse im einzelnen nicht ausgeführte Kette, der $U$ als cin Glied angehört, bildet die psychische Grundlage für $X$ 's Reaktion auf die äußeren Ereignisse. Die Abbildung A zeigt weiters keinc Handlungen; aber sie demonstriert schon den springenden Punkt, nämlich daß eine zweite Kausalkette cxistiert, die den erläuterten Zirkel auslöst und dic $A$ blcitung des Gesamtnutzens von $A$ aus dem intrinsischen Nutzen von $S$ vereitelt.

Die $\Lambda$ bbildung $\mathrm{B}$ berücksichtigt nun ausdrücklich auch Handlungen; sie paß3t auf das Schwangerschaftsbeispiel, wobei $A$ für die Schwangerschaft der Frau steht, $S$ für ihre Freude und $H$ etwa für die Handlung, Verhütungsmittel abzusetzen. Hier laufen nun von $U$ zu $S$ tatsächlich z.wei Kausalketten, die erste über $H$ und $A$, dic zweite wie in der Abbildung A. Wie bereits erläutert, stellt die erste kcin Problem dar, weil der kausale Einfluß von $I J$ auf $S$ in dieser Kette von $H$ abgeschirmt wird; daher kann hier der extrinsische Nutzen etwa von $H$ aus dem Gesamtnutzen von $A$ (und $\sim A$ ) abgeleitet werden. Dagegen ist es auch hier wieder dic zweite Kausalkette - die nicht durch die äußere Welt abgeschirmtc - die dafür verantwortlich ist, daß der Gesamtnutzen von $A$, und folglich auch $\operatorname{der}$ von $H$, nicht aus dem Nutzen von $S$ abgeleitet werden kann.

Der intuitive Kern von alledem ist, daß die praktische Überlegung nicht von der Stelle kommt, wenn sie einzig vom Wunsch nach Glück ausgeht. Wenn man sich z. B. für einen Beruf zu entscheiden hat, so kommt man zu keinem Ergebnis, wenn man nur danach fragt, wie befriedigend wohl die verschiedenen Optionen wären. Der Grund dafür liegt nicht darin, daß die Entscheidung so komplex wäre, sondern darin, daß die Optionen an sich kein bestimmtes Befriedigungspotential haben. Vielmehr muß man bereits zumindest eine grobe Vorstellung davon haben, wie sehr man die jeweiligen Berufe ausüben will; erst dann kann man sich sinnvoll fragen, wie sehr sie jeweils befriedigen würden. Das war der wesentliche Punkt an Joseph Butlers vor über 250 Jahren geübten Kritik am Hedonismus:

„The very idea of an intcrested pursuit necessarily presupposes particular passions or appetites, since the very idea of interest or happiness consists in this, that an appetite or affection enjoys its object. It is not because we love ourselves that we find delight in such and such objects, but because we have particular 
affections towards them. Take away these affections, and you lcave self-love absolutely nothing at all to employ itself about; no end or object for it to pursuc, excepting only that of avoiding pain." (Butler, 1729, Preface, \31)

Unsere Thesen (A) und (B) übersetzen dies, wenn man so will, in unsere Begrifflichkeit, und sie weisen auf eine wichtige Unvollständigkeit der Entscheidungstheorie hin. Die Lücke muß sich nicht in jedem Fall auftun, in dem satisfaktive Zustände in die praktischc Überlegung cinbezogen sind. Manchmal läßt sich der Überlegungszirkel unterdrücken, z. B. durch überwiegende moralische Gesichtspunkte; ein andermal mag der Finfluß der inneren Kausalkette vernachlässigbar sein; und natürlich wäre es interessant, sich diese Ausnahmen genauer anzusehen. In vielen, ganz gewöhnlichen Fällen jedoch bleibt der Zirkel virulent.

\section{Wie man dem Zirkel nicht entgeht}

Obwohl die hier aufgeworfene Schwierigkeit für die praktische Überlegung etwas konstruiert aussehen mag, läßt sie sich nicht so einfach dekonstruieren. Jedenfalls bietet keiner der folgenden fünf Einsprüche einen Ausweg.

(I) Man könnte denken, daß wir uns zu stark auf das intuitive kausale Bild, auf das sich die praktische Überlegung bezieht, und dessen formaler Entsprechung in Gestalt von Entscheidungsbäumen gestützt haben. Und man könnte meinen, daß der Zirkel in einer geeigneten anderen Formalisierung der Entscheidungstheorie verschwindet. Das ist wenig plausibel, wenn diese andere Formalisierung eine der geläufigen Versionen der kausalen Entscheidungstheorie sein soll, da diese Vcrsionen dieselben Wurzeln haben, auf die auch wir uns bezogen habcn. Stattdessen könnte man auf die sogenanntc ,evidentielle Entscheidungstheoric ${ }^{6}$ verweisen; ${ }^{17}$ doch bezweifeln wir, daß dies hier hilfreich ist. Jedenfalls liegt die Beweislast vorerst nicht bei uns.

(II) Man könnte denken, daß es sich hier einfach um eine Neuauflage einer alten Widerlegung des Hedonismus handelt, wie ihn heutzutage sowieso niemand mehr vertritt, und daß wir mithin off cne Türen einrennen. Das wäre allerdings ein Mißverständnis; das aufgewiesene Problem ist ein allgemeineres. Unsere Kritik betrifft nicht nur die hedonistische Behauptung, daß einzig und allein satisfaktive Zustände intrinsisch evaluiert sind. Vielmehr geht es uns um die entscheidungstheoretische Kon-

17 Wie sie etwa Eells (1982) vertritt. 
zeption der Herleitung extrinsischer Werte aus intrinsischen; und dazu benötigen wir nur die vicl schwächere Annahme, daß zumindest einige dcr intrinsisch evaluierten Sachverhalte cigene innere Zustände sind. Nicht bloß der Hedonist, sondern jeder, der diese Konzeption und dicse Annahme teilt, kommt in dic beschriebene Schwierigkeit.

Trotzdem, so könnte man sagen, verdankt sich diese Schwierigkeit dem hedonistischen Rahmen, d. h. dem Versuch, den cxtrinsischen Nutzen äußerer Sachverhalte vom intrinsischen Nutzen innerer Zuständc ableiten zu wollen. Könnte man nicht einfach dicsen Versuch bleibenlassen? Das wäre sicher keine Lösung. Denn schließlich sind die Nutzenwertc äußerer Sachvcrhalte irgendwie mit den intrinsischen Nutzenwerten inncrer $\mathrm{Zu}$ stände vcrknüpft, auch wenn es sich dabei um keine entscheidungstheoretische Ableitung handelt. Dieser Verknüpfung gilt es Rechnung zu tragen, anstatt sie zu leugnen.

(III) Man könntc weiterhin denken, daß sich die Schwierigkeit einer doppeldeutigen Verwendung des Nutzenbegriffs verdankt. So könnte man insbesondere meinen, $\mathrm{da} \beta$ in Abbildung $\mathrm{A}$ der für $S$ kausal relevante Nutzen von $A$ - nennen wir ihn $U^{k}(A)$ - nicht derselbe ist wie jener, der aus $S$ hergeleitet wird. Worin besteht $U^{k}(A)$ ? Ist er wirklich, wie behauptet, mit $U^{\prime}(A)$ identisch?

Man könntc meinen, $\operatorname{da} \beta L^{k}(A)$ eine andere Art von Evaluation ist als jene, die in die praktische Überlegung Fingang findet. Nicht unbedingt. Wir wollen nicht leugnen, daß sich Sachverhalte auch in anderer, z. B. ästhetischer Hinsicht evaluiercn lassen und daß solche Evaluationen für satisfaktivc Zustände kausal relevant sein können. Das ändert aber nichts daran, daß auch dic in der praktischen Überlegung verwendete Art der Fvaluation dicsc kausale Relevanz besitzt; genau das zeigten ja all unsere Beispicle. (Das gilt selbst für das Fußballbeispicl, wo die Wünsche der bciden Männcr keine praktische Relevanz haben, weil sic für das von ihnen jeweils favorisierte Team nichts tun können; doch würden sie das, wenn sie es könnten.)

Doch könnte man immer noch einwenden, daß es für $U^{k}(A)$ drei verschiedene Kandidaten gibt, nämlich den intrinsischen, den extrinsischen und den Gesamtnutzen von $A . U^{k}(A)$ könnte mit $U^{i}(A)$ identisch sein; dann ergäbe sich allerdings kcin Überlegungszirkel, weil $U^{i}(\Lambda)$ nicht abgeleitet und daher unabhängig gegeben ist. Mit $U^{c}(A)$ hingcgen kann $U^{k}(A)$ nicht identisch sein; denn dann ließe sich unter den Bedingungen des Zirkels überhaupt kein extrinsischer Nutzen von $A$ bestimmen. Schließlich kann $U^{k}(A)$ anscheinend auch nicht mit $U^{\prime}(A)$ identisch scin; $\operatorname{denn} U^{\prime}(A)$ war als Summe von $U^{i}(A)$ und $U^{i}(A)$ erklärt, dic, 
wenn $U^{\prime}(A)$ nicht existiert, ebenfalls nicht existicren kann. Demnach, so dieser Einwand, ist es völlig mysteriös, welcher Nutzen diesen Zirkel erzeugen soll.

Die formale Entgegnung darauf lautet, daß im Abschnitt 1 in Vorweg. nahme dieses Finwands betont wurde, daß es nur eine Annahme und keine Definition ist, wenn $U^{\prime}(A)$ gleich der Summe von $U^{i}(A)$ und $U^{\prime}(A)$ gesetzt wird. Läßt man diese Annahme fallen, wie es der Zirkel erzwingt, fällt der letzte Schritt des Einwands weg, so daß der Gleichsetzung von $U^{k}(A)$ mit $U^{t}(A)$ nichts im Wege steht.

Aus unseren Beispielen ergibt sich aber auch eine intuitive Entgegnung. Die Freude der Frau hängt davon ab, wie sehr sie sich das Baby wünscht; meine berufliche Befriedigung ist dadurch bedingt, wie sehr ich den Beruf, den ich habe, auszuüben wünsche. In diesen Fällen ist der die Befriedigung beeinflussende Wunsch nicht (oder nicht nur) ein intrinsischer. Im Falle der Frau ist cs sozusagen ihr Gesamtwunsch, der sich aus vielen Quellen speist: aus dem intrinsischen Wunsch nach einem Kind, aus dem negativen Nutzen der Kosten, die das Kind verursacht; aus dem Wunsch, ihren Mann oder sogar ihre Fitern zufriedenzustellen, etc. und natürlich aus ihrer Freude an dem Kind. Im Falle des Berufes ist es sogar leicht vorstellbar, daß er überhaupt nicht intrinsisch gewünscht wird, sondern nur als Mittel zur Befricdigung, zum Geldverdienen, etc.

Aus diesem Einwand ergibt sich also nicht, daß unsere intuitive Beschreibung des Zirkels falsch ist; vielmehr folgt, daß mit der üblichen Eintcilung des Nutzens in intrinsischen, extrinsischen und Gesamtnutzen etwas nicht stimmt. Die Beispiele weisen auf eine Art von Gesamtnutzen hin, der unter den Bedingungen des Zirkels weder intrinsisch noch entscheidungsthcoretisch ableitbar ist. $\mathrm{Zu}$ suchen ist mithin eine adäquatere Klassifikation des Nutzens.

(IV) Man könnte immer noch den Eindruck haben, daß sich unser Problem einem unklaren Nutzenbegriff verdankt. Wir haben uns nämlich massiv auf die kausale Trennbarkeit von Nutzen und Befriedigung gestützt; und diese mag anfechtbar erschcinen. Tatsächlich wird beides vielfach gleichgesetzt. Diese Gleichsetzung wollen wir nun nach zwei Richtungen hin diskutieren, wobei wir insgesamt auf folgendes hinauswollen: Wenn man (a) Nutzen auf Befriedigung reduziert, wird man dem Nutzenbegriff nicht gerecht; reduziert man umgekehrt (b) Befriedigung auf Nutzen, so wird man dem Phänomen der Befriedigung nicht gerecht. Schließlich gibt es (c) eine Idee zur Überbrückung der Differenz von Nutzen und Befriedigung, die uns attraktiv erscheint; doch bringt sie, wie sich zeigen wird, 
keine Lösung des Überlegungszirkels. Betrachten wir (a), (b) und (c) nähcr.

(IVa) Der Handelnde ist nach der Entscheidungstheorie ein höchst eigenartiges Wescn. Er ist dauernd damit beschäf tigt, seinen Nutzen zu berechnen und seinen erwarteten Nutzen zu maximieren - und damit hat es sich. Aber hat er jemals etwas von all der Mühsal? Was ist am Fnde seine ,Auszahlung? In den Lehrbuchbeispielen, wo Geld vorläufig die Rolle des Nutzens übernimmt, ist die $\Lambda$ ntwort einfach: Er bekommt eben Geld. Aber in Wirklichkeit lassen sich Geld und Nutzen nicht so einfach gleichsetzen. Was bckommt er dann? Die natürliche Antwort darauf ist, daß er dann eben den Nutzen bekommt; schließlich liegt kein Sinn darin, den Erwartungswert einer Quantität zu maximieren, von der man nichts bekommt. So wcit ist der Nutzen allerdings nichts weiter als ein Namc für das, was der Handelnde am Ende bekommt. Aber nun wissen wir doch, worin seine ,Auszahlung' besteht; sie besteht darin, wie er sich fühlt und wie es ihm geht, d. h. in unserer Terminologie, in seinem satisfaktiven Zustand, gcmessen auf irgendeiner Skala, wie sie schon im alten I ustkalkül konzipiert war. Das also ist sein Nutzen.

So sieht kurz die Überlegung aus, die Nutzen zwanglos auf Befriedigung reduziert; viele haben sich von ihr verführen lassen. ${ }^{18}$ Doch sic ist noch unzureichend. Denn so weit besagt sie bloß, daß die zukünftigc Auszahlung des I Iandelnden in seinen zukünftigen satisfaktiven Zuständen bestehen wird. Wie aber ist die gegenwärtige Nutzenfunktion zu verstehen? Es scheint nur eine Möglichkeit zu geben: Der gegenwärtige Nutzen eines möglichen rukünftigen satisfaktiven Zustands ist einfach der Grad der Bcfriedigung, die mit ihm eintreten würde, und der gegenwärtigc Nutzen anderer Sachverhalte besteht in ihrer erwarteten Befriedigung, d. h. er ist ein Erwartungswert, der ausdrückt, für wie befriedigungsfördernd diese Sachverhalte gehalten werden.

Diese Konzeption ist jedoch immer noch unzureichend. Denn das von ihr gezcichnete Bild praktischen Überlegens und rationalen Handelns enthält nur zukünftigc Befriedigung und gegenwärtige Über»eugungen darüber; die W'ünsche oder Ziele des Handelnden kommen in ihm nicht vor. Es scheint uns klar, daß ein solches Bild grundlegend vcrzerrt sein muß.

in Diese Reduktion ist für den klassischen Utilitarismus charakteristisch, doch findet sie sich auch in der modernen Wohlfahrtstheorie. Vgl. Bentham (1970), Kap. I, Mill (1976), Kap. 2, Sidgwick (1874), Buch II, und Pigou (1920). Vgl. auch Sen (1980/81), Teil 2, und Griftin (1986), Kap. 1. 
Man könnte es nachzubessern versuchen, indern man die Wünsche des Handelnden innerhalb dieses Bildes definiert. Das erscheint jedoch von vornherein wenig aussichtsreich, da Wünsche gemeinhin als eine grundlegende Art propositionaler Einstellungen gelten, die sich nicht auf andere propositionale Einstellungen reduzieren lassen. ${ }^{19}$ Außerdem könnte man sie wohl nur dadurch ins Bild aufnehmen, daß man den bereits definierten gegenwärtigen Nutzen des Handelnden zugleich als Repräsentation seiner mehr oder weniger starken Wünsche auffaßt. Dieses Vorgehen würde jedoch den Hedonismus in eine Wahrheit a priori verwandeln; denn es postuliert, daß sich die Wünsche des Handelnden intrinsisch ausschließlich auf satisfaktive Zustände richten. Außerdem ergibt sich ein bekanntes Problem des Hedonismus: Um den intuitiven Wunschbegriff mit diesem Postulat in Einklang zu bringen, muß jedweder Wunsch mit einer Befriedigungserfahrung und jedwede Präferenz mit ciner Differenz in der (erwarteten) Erfahrung von Befriedigung in Zusammenhang gebracht werden. All das erscheint nicht minder verzerrt.

Der Punkt ist mithin schlicht der, daß gegenwärtige Wünsche nicht mit gegenwärtigen Erwartungen über zukünftige satisfaktive Zustände gleichgesetzt werden können und daß der gegenwärtige Nutzen cben nicht die letzteren, sondern die ersteren repräsentieren muß, wenn er im praktischen Überlegen und im rationalen Handeln die ihm von der Fntscheidungstheorie zugedachte Rolle spielen soll. ${ }^{20}$

Schließlich ist darauf hinzuweisen, daß die zur Diskussion stehende Konzeption des Nutzens den Uberlegungszirkel nicht zum Verschwinden bringt, sondern bloß transformiert. Denn unsere Thesc (B) besagte dann, $\mathrm{da} ß$ die gegenwärtige Nutzenerwartung des Handelnden für seine zukünftige Befriedigung kausal relevant ist; und dafür wären genau dieselben Beispiele einschlägig. Einfacher und nicht probabilistisch ausgedrückt bedeutet das, daß die gegenwärtige Überzeugung darüber, daß dieser Sachvcrhalt zu jener Befriedigung führen wird, kausal dafür relevant ist, ob das tatsächlich der Fall ist. Diese Übcrzcugung würde sich also sclbst bewahrheiten oder jedenfalls ihre eigene Wahrheit kausal affizieren. Und daraus entstünde dann ein analoger Cuberlegungszirkel: Um zu wissen, welche zukünftige Befriedigung man erwarten soll, sollte man bereits rissen, welche Erwartung man diesbezüglich hat. Diese Konzeption des

19 V'gl. auch Lewis (1988), der ein allgemeines Argument dafür bringt, daß sich W'ünsche nicht auf ('berzeugungen reduzieren lassen.

* Dies wird in Kusser (1989), Abschn. 3.3, ausführlicher begründet. 
Nutzens ist also, um ein Fazit zu ziehen, nicht nur fehlgeleitet, sie löst auch nichts - Gründe genug, um von ihr Abstand nehmen. ${ }^{21}$

(IVb) Tatsächlich ist die Geschichte des Nutzenbegriffs in diesem Jahrhundert durch diesen Abstand geprägt, wie er sich vor allem in der Theorie der ,revcaled preferences' zeigt. $^{22}$ Die meisten Theoretiker und insbesondere die Ökonomen begreifen heute Nutzenfunktionen als Repräsentation der unterschiedlich starken Wünsche, wie sie sich in den Präferenzen und den Handlungen rationaler Personen manifestieren. ${ }^{23}$ Dann stellt sich jedoch erneut die Frage nach dem Verhältnis von Nutzen und Befriedigung.

Da gibt es nun die zähe Neigung, die soeben diskutierte Antwort umzudrehen und zu sagen, daß Befriedigung eben das ist, was sich einstellt, wenn Wünsche in Frfüllung gehen - womit der Befriedigungsbegriff zu einem rein formalen wird. ${ }^{24}$ Das klingt absurd, und vermutlich hat es deshalb niemand so deutlich gesagt. Doch hat die Neigung, Befriedigung (oder Lust oder Glück) so zu konzipieren, eine reiche Geschichte. Kant ctwa sagt:

"Glückseligkeit ist der Zustand eines vernünftigen Wesens in der Welt, dem es im Ganzen seincr Existenz alles nach Wunsch und Willen geht." ${ }^{25}$

Und Rawls schreibt:

21 F.s ist anzumerken, daß der von G. S. Becker und K. Lancaster u. a. entwickelte und vor allem unter Okonomen prominente Ansatz, auch Haushalten Produktionsfunktionen zuzuordnen (vgl. Michael/Becker, 1973), nicht minder von unserer Schwierigkeit betruffen ist. Im Rahmen dieses Ansatzes wird der Konsument als Produzent von Nutzen oder Lust uder Befriedigung angesehen, was die Möglichkeit eröffnet, die Produktionstheorie auf den Konsumenten anzuwenden. Unser Zirkel implizier, daß die Produkt ionsfunktion des Konsumenten für ein gewisses Grundgut (d. h. eine bestimmte Art von Befriedigung) in der Regel ein zusäezliches Argument enthalten muß, nämlich die Stärke seines Wunsches nach denjenigen Gütern, aus denen er das Grundgut produziert; und nach dem gerade diskutierten verzerrten Bild wäre ein anderes Argument zusätzlich zu berücksichtigen, nämlich die t'berzeugungen darüber, wieviel vom Grundgut aus welchen Mengen anderer Güter produziert werden kann. So oder so ergibt sich daraus eine Schwierigkcit oder Zirkularität, die in der herkömmlichen Produktionstheorie nicht entsteht und nicht behandelt wird.

22 Die Gründungsväter dieser Theorie sind v. Neumann/Morgenstern (1944), Abschn. 1.3, und Samuelson (1947), Kap. IV.

23 Positivistischer Gesinnte halten in der Tat nur Handlungen und Präferenzen für real und reden so, als ob Wunschstärken und Nutzenwerte nur Als-ob-Konstruktionen wären. Fin solcher Operationalismus gilt aber in der gegenwärtigen Wissenschaftstheorie als überholt; vgl. auch Sen (1973), Abschn. 2.

24 Dem entspricht ein rein formaler Begriff der Auszahlung als eines Nuzzens, den man immer dann schon hat, wenn ein Wunsch in Erfüllung geht. Vyl. Griffin (1986), Kap. 1, und Sen (1980/81), Teil 4.

25 Kant (1788), S. 224. 
„... we are happy when our rational plans are going well, our more important aims being fulfilled, and we are with reason quite sure that our good fortune will continuc." $" 26$

Gleichwohl sollte man dieser Neigung nicht nachgcben. Zu häufig war man nicht befriedigt, obschon man bekommen hat, was man wollte, und zu lebhaft und eigenständig ist die Phänomenologie der satisfaktiven Zustände, als daß dic begrifflichc Verschmelzung von Wunscherfüllung und Befriedigung durchführbar wäre.

Natürlich gibt es Gründc für diese Neigung. Einer ist sicherlich, daß es zumindest die Regel ist, daß Wunscherfüllung bef riedigt; doch es gibt Ausnahmen. Ein besserer Grund ist, da $\beta$ wir nicht umhinkönnen, jeweils anzunehmen, daß die Erfüllung unserer $W$ ünsche uns befriedigen wird. Sind wir der Überzeugung, daß die Erfüllung eines $W^{\prime} u n s c h e s$ uns nicht befriedigen wird, und halten wir gleichwohl an diesem Wunsch fest, so haben wir entweder überwiegende, z. B. moralische Gründe dafür, oder wir sind einfach töricht. Die Situation ähnelt Moores Paradox: Wir müssen jeweils glauben, daß das, was wir glauben, wahr ist; doch wissen es andere vicllcicht besser und wir später womöglich auch. Genauso müssen wir jeweils glauben, daß das, was wir wünschen, uns befriedigt; aber andere wissen es möglicherweise besser, und wir merken es vielleicht auch noch. Diese Gründe mögen die diskutierte Neigung genährt haben; doch ist sie dadurch nicht gerechtfertigt.

(IVc) Hat man erst einmal eingeräumt, daß der Zusammenhang zwischen Befriedigung und Wünschen oder Nutzen kontingent ist, so kommt man um den Zirkel nicht mehr herum. Zwar wird immer wieder gesagt, daß unsere wirklichen oder wahren Wünsche jene seien, deren Verwirklichung befriedigt, und daß wir im Idcalfall dicse Wünsche hätten. ${ }^{27}$ Wir teilen diese Ansicht und werden unten darauf zurückkommen. Der Überlegungszirkel ist davon allerdings nicht betroffen, da er auf dem tatsächlichen Zusammenhang zwischen Wünschen und Befricdigung beruht und nicht durch die Betrachtung des Idealfalls verschwindet.

(V) Gleichwohl mögen unsere Zirkclthesen immer noch ein ungutes Gefühl zurückgelassen haben, das vielleicht im folgenden Einwand zum

26 Rawls (1971), S. 548.

7. Vyl. etwa Russell (1921), Kap. III, insbes. S. 72 ff, Gosling (1969), Kap. 7, Tugendhat (1984), S. 45 f, und Trersky/Kahneman (1986), S. 138. - Von einer rationalen Person ist nicht zu verlangen, daß sie wahre Wünsche hat, genauso wenig, wie sie wahre Übcrzeugungen haben muß. Zur Rationalität gehört nicht, Wissen zu haben, sondern Wissen zu suchen (wobei natürlich das erste häufig Resultat des zweiten ist). Ebenso gehör es sicherlich zur Rationalität, nach wahren Wünschen zu streben. Viclleicht ist dieser Sinn von Rationalität in den obigen Zitaten son Kant und Rawls intendiert. 
Ausdruck kommt: Nach der Thesc (B) soll sehr häufig etwas eine Person befriedigen, weil sie es gewünscht hat, und darin soll dann irgendwic ein Problem liegen. Fragt man aber die Person, warum sie die Sache wünscht, so ist die natürliche Antwort: „Weil es mich befriedigt!“ Und an diescr Antwort ist nichts auszusetzen - außer, daß offenbar nicht bcides, diese $\Lambda$ ntwort und die These (B), richtig sein kann.

Dieser Einwand kann einen leicht verwirren. Doch besteht hier kein Widerspruch; das "wcil“" wird dabei in zwei verschicdenen Bedcutungen verwandt. Sagt man, etwas befriedige eine Person, weil sie es wünsche, so redet man über eine der Ursachen ihrer Befriedigung; sagt sie jedoch, sie wünsche es, weil cs sie befriedigc, so spricht sie über einen ihrer praktischen Gründe für ihren Wunsch.

Ganz so lcicht ist dic Sache aber nicht abgetan; es gibt hier noch zwei Probleme:

(Va) Wir haben gerade zugcgeben, daß die Überzeugung, daß etwas befriedigen wird, cin praktischer Grund dafür ist, cs zu wünschen. Was aber ist ein praktischer Grund? lst $A$ nicht genau dann ein praktischer Grund für $B$, wenn $A$ in einer zu $B$ führenden praktischen Überlegung, d. h. also in einer zu $B$ führenden entscheidungstheorctischen $\Lambda$ bleitung wesentlich verwandt wird? Doch enthält der letzte Satz zwei verschiedene, durch das „d. h.“ zu unrecht gleichgesetzte Erläuterungen. Die erste ist vage und richtig, die zweite präzis und falsch. Man wird also nicht umhinkommen, zwei Arten praktischer Gründc anzuerkennen: die in der Entscheidungstheoric explizierten und noch andere; und zu dieser anderen Art gehört offenbar die Überzeugung, daß etwas befriedigen wird. Es bleibt die Aufgabe, diese Art von praktischen Gründen genaucr zu erklären.

(Vb) Die Kausalisten unter den Handlungstheoretikcrn, denen wir uns, wie klar sein dürfte, angeschlossen haben, behaupten, daß sich bei einer rationalen Person das, was aus ihrer Innenperspektive ein praktischer Grund ist, aus der Außenperspektive als eine Ursache darstellt. Da wir einräumen, daß die Überzeugung, daß etwas befriedigen wird, für eine rationale Person ein Grund ist, es zu wünschen, müssen wir damit auch zugcben, daß diese Überzeugung ihren Wunsch verursacht. Da wir außerdem bchauptet haben, daß ihr Wunsch für ihre Befriedigung kausal relcvant ist, folgt daraus (mit der Transitivität kausaler Relevanz), daß die Überzeugung, daß etwas befriedigen wird, für ihre eigene Wahrheit kausal relevant ist. Soll man diese Folgerung wirklich akzeptieren? ${ }^{28}$

28 Diese Problemliste ist natürlich in keiner Weise erschöpfend. Eine wichtige, wenn auch 


\section{Wie man dem Zirkel gerecht werden kann}

So weit scheint der Zirkel ungebrochen zu sein, doch hat die Diskussion etliche offene Fragen aufgeworfen. Ist der Zirkel wichtig, so fordert er eine ganze Theorie. Die haben wir nicht, aber einige konstruktive Beobachtungen und positive Schlußfolgerungen möchten wir schließlich noch anbieten.

Bereits im Abschnitt 1 haben wir auf die Rudimente einer formalen I.ösung des Problems hingewiesen. Betrachten wir, um sie etwas zu verdeutlichen, noch einmal die Formeln (1) und (2):

$$
\begin{aligned}
& U^{\prime}(A)=U^{\prime}(B) P(B \mid A)+U^{\prime}(\sim B) P(\sim B \mid A), \\
& U^{\prime}(A)=U^{\prime}(A)+U^{\prime}(A) .
\end{aligned}
$$

In ihnen ist sozusagen ein reguläres Verhältnis von $A$ und $B$ unterstellt. Im zirkulären Fall, der, wie gesagt, nur dann eintritt, wenn $B$ für einen zukünftigen satisfaktiven Zustand steht, sind die kausalen Überzeugungen der Handelnden allerdings nicht durch $P(B \mid A)$ zu repräsentieren, sondern durch $P\left(B \mid A \& U^{\prime}(A)=x\right)$ mit einem geeignet gewählten Wert von $x$. Also sollte man in (1) die erste Wahrscheinlichkeit durch die zweite ersetzen. Wenn nun bekannt wäre, $\operatorname{da} \beta U^{\prime}(A)$ den Wert $x$ annimmt, so ergibt sich aus den modifizierten Formeln (1) und (2) ein Wert $y$ für $U^{t}(A)$. Das heißt, wenn $\operatorname{der}$ Wert von $U^{t}(A) x$ ist, so sollte er $y$ sein, was nur erfüllbar ist, wenn $x=y$ ist. Mit anderen Worten: Nur L.ösungen der Gleichung

$$
\begin{aligned}
x & =U^{\prime}(A)+U^{\prime}\left(B \mid A \& U^{\prime}(A)=x\right) \\
& +U^{\prime}(\sim B) P\left(\sim B \mid A \& U^{\prime}(A)=x\right)
\end{aligned}
$$

ganz andere Variation des Einwands (IV) besagt, daß wir von einer problematischen Trennung in den Gegenständen oder Inhalten von Wünsehen ausgehen. In der Tat haben wir implizit angenummen, daß es $z$. B. über den Wunsch nach einem bestimmten Bordeaux hinaus noch den Wunsch nach dem Geschmack dieses Bordeaux gibt, d. h. allgemein, daß der Inhalt eines Wunsches in zwei Teile zerlegt worden kann, einen externen Teil, der sich auf äußere Fireignisse bezieht, und einen internen, der nur von inneren Frfahrungen handelt. Dagegen könnte man cinwenden, daß diese Trennung unzulässig sei, weil der Inhalt eines Wunsches im Prinzip beschreibbar sein müsse und weil satisfaktive Zustände sich oftmals nur unter Bezugnahme auf die äußere Wielt beschreiben lassen (z. B. auf einen bestimmten Jahrgang einer bestimmten Weinlage). Und man könnte ferner vermuten, daß wir den Zirkel genau aus dieser Trennung gewinnen, indem wir einen Wunsch in zwei aufspalten und dann aus dem Z,usammenhang der beiden Hälften ein Problem kunstruieren. Demgegenüber halten wir diese Trennung für legitim; dies zu verteidigen, würde uns freilich (zu) weit in die Philosophie des Geistes und der Sprache abführen - d. h. genauer, in die Debatte um den Antiindividualismus von Burge (1979), wo in Bezug auf Ubeneugungen analoge Fragen diskutiert werden. 
sind zulässige W'erte von $U^{\prime}(A)$; andernfalls entspricht der Gesamtnutzcn von $A$ nicht dem Erwartungswert der Befriedigung, den $A$ in Bezug auf $B$ unter der $\Lambda$ nnahme dieses Gesamtnutzens hat.

In der Tatsache, daß die Unbekannte $x$ auf der linken und der rcchten Seitc der Gleichung (3) erscheint, spiegelt sich noch einmal unmittelbar der ¿̌berlegungszirkel. Man beachte, da $\ \Lambda$ nun kein extrinsischer Nut\%en mehr zugcschrieben wird; wenn der Gesamtnutzen der Formel (3) gehorcht, so läßt er sich nicht mehr als Summe von intrinsischem und extrinsischem Nutzen verstehen. Die Gleichung (3) hat keine, genau cine oder, was häufig der Fall sein wird, mehrere Lösungen, je nachdem wie dic bedingten Wahrscheinlichkeiten in (3) mit $x$ variicren. Wenn das mathematische Problem sauber formalisiert wäre, könnte man darauf wie in der Gleichgewichtstheorie Fixpunktthcoreme anwenden. Wir sind dem aber nicht nachgegangen, aus Gründen, die weiter unten zur Sprache kommen.

Gleichwohl ist der in (3) zum Ausdruck kommende Gleichgewichtsgedanke intuitiv sehr hilfrcich; sechs offen gebliebene Fragen lassen sich auf seiner Grundlage informell klären.

Vier Arten von Nutzen: Die Gleichung (3) stellt zwischen dem Nutzen satisfaktiver 7ustände und dem äußerer Sachverhalte einen Zusammenhang her, wie wir ihn im 'Teil (II) des vorigen Abschnitts gesucht haben. Diescr Zusammenhang crgänzt das entscheidungstheoretische Bild praktischer Rationalität. Innerhalb des altes Bildes gibt es nur zwei $\Lambda$ rten von Nutzen: intrinsische Nutzenwerte, von denen alle Ableitungen ausgehen, und abgelcitete, d. h. extrinsische und Gcsamtnutzen werte. In (3) hingegen sind die Rollen von Nutzenwerten anders vertcilt. Man kann nicht sagen, $\mathrm{da} \beta$ in (3) $U^{\prime}(A)$ aus $U^{\prime}(B)$ odcr $U^{i}(B)$ abgeleitet wäre. ${ }^{29}$ Das ist besonders deutlich, wenn (3) mehr als einc Lösung hat. Dcr intrinsische Nutzen satisfaktiver Zustände, wie $B$ ciner ist, dient also in der Regel nicht als Prämisse einer Ableitung. Entsprechend ist der Nutzen vieler äußerer Sachvcrhalte weder abgeleitet noch im ursprünglichen Sinn intrinsisch. Vielmchr kann man das Verhältnis zwischen $U^{\prime}(B) \operatorname{oder} U^{i}(B)$ und $U^{\prime}(A)$ in (3) als eines der Kontrolle bezeichnen. Der Nutzen äußercr Sachverhaltc wird in dem Sinne von dem Nutzen satisfaktiver Zustände kontrolliert, daß der erstere mit dem letzteren im Gleichgewicht sein muß

29 W'enn $B$, wic in (3), ein satisfaktiver Zustand ist, so werden $U^{\prime}(B)$ und $U^{\prime \prime}(B)$ gewöhnlich zusammenfallen. 
und $z u$ verändern ist, sobald ein Ungleichgewicht entstanden ist. ${ }^{30}$ Nutzenfunktionen können mithin auch einer solchen Gleichgewichtskontrolle unterliegen; das ist die wesentliche I,ehre, dic aus dem Überlegungszirkel zu ziehen ist.

In diesem Sinn crgcben sich aus (3) zwei neue Arten von Nutzenwerten: kontrollierende und kontrollierte. Damit klärt sich auch die in (III) aufgetretene Verwirrung darübcr, welcher Art der für Befriedigung kausal rclcvantc Nutzen $U^{k}(A)$ von $A$ ist. Es ist der unabgeleitetc Gesamtnutzen von $A$ - wobei "unabgelcitet“ nun nicht mehr „intrinsisch“ im alten Sinn und „Gesamtnutzen“ nicht „abgeleitct“ bedeutet. Darin liegt nichts Paradoxes, wenn man nur einsieht, daß es diese vier Arten von Nutzen gibt. $^{31}$

Zwei Arten praktischer Gründe: Das eben Gesagte beantwortet auch die Frage in (Va) des vorstehenden Abschnitts. Es gibt tatsächlich zwci Arten praktischer Gründc: jene, die als Prämissen in entscheidungstheoretischen Ableitungen fungieren, und jene, die Gleichgewichte wie die Lösungen von (3) stützen. Der Wunsch nach Befriedigung und der Glaube, daß etwas befriedigen wird, oder, allgemeincr, Nutzen und Wahrscheinlichkeiten wie diejenigen auf der rechten Seite von (3) sind Gründe dieser zweiten Art. Auf die Frage, warum man etwas wünscht, mit „weil es mich befriedigt“" zu antworten, ist also völlig richtig. Falsch wäre nur die Annahme, daß dieser Wunsch damit aus dem Wunsch nach Befriedigung abgeleitet ist.

*) Tatsächlich ist dieses Vcrhältnis weniger einseitig, als das Wort „Kontrolle“ andeutet. Frlebt man z. B., daß eine gewisse Sache nicht mchr in der früheren W'cise befricdigt, so muß man nicht unbedingt den Wunsch nach dieser Sache aufgeben; man kann auch versuchen, sie in ncuer W'cise schätzen zu lerncn, d. h. sich so zu verändern, daß man einc anderc Hefriedigung aus ihr zicht - indem man z. B. seinc Aufmerksamkeit auf anderes lenkt, die alten Aren von Befriedigung geringer schätzt, usw. Man kann also ein Gleichgewichı auch dadurch wieclerherstellen, daß man satisfaktive Zustände neu evaluiert bzw. sich dahin bringt, daß die gleichen äuleren Ereignisse andere satisfaktive Zustände hervorrufen. (Letzteres ist in der 'Tat die angemessenere Beschreibungsweise, da ein satisfaktiver Zustand durch seine Evaluation wesentlich charakterisien ist und mit einer neuen Evaluation cin anderer wird; eine nicht mehr bloß positiv bewertete Errcgung etwa ist nicht mehr die alte Errcgung). Dicse Anpassung der Frcuden an die W'ünsche (anstatt der Wünsche an die Freuden) ist tatsächlich häufig zu finden; man sollte sie nicht als irrational denunzieren.

"Hierbei haben wir nur die sozusagen reinen Arten von Nutzen erwähnt. Sie können sich auch mischen, und dann wird es wirklich kompliziert. So mag z. B. für die schwangere Frau das Kind, das sie erwartet, alle drei Arten von Nutzen haben: einen intrinsischen, weil sie neues Leben positiv bewcrtet; aus vielerlei Gründen einen extrinsischen Nutzen, etwa weil es viel Gcld kosten wird; und schließlich noch jener von ihrem Wunsch, glücklich zu scin, kontrollierte Nutzen. 
In gleicher Weisc ist die Behauptung zu qualifizieren, daß das, was aus der Inncnperspektive ein praktischer Grund ist, von außen betrachtct cine Ursache ist. Diese Behauptung läßt sich nur für die entscheidungstheorctisch funktionierenden Gründe, für die sie ja auch intendiert war, aufrechterhalten. Die equilibristischen praktischen Gründe verursachen hingegen nur, daß man an einem durch sic gestützten Wunsch festhält, nicht jedoch seinen Erwerb. Auf diese Weise läßt sich dic unerfreuliche Schlußfolgcrung aus (Vb) vermeiden. Gleichwohl müssen wir uns diesen Punkt noch genauer anschauen.

Der Status der Überzeugung, daß die Verwirklichung eines $W$ unsches befriedigen wird: Nach dem eben Gesagten beglcitet diese Überzeugung den Wunsch ehcr, als daß sie ihm vorausgeht. In der Tat begleitet sic ihn notwendig; gibt cs keine anderweitigen Gründe für einen Wunsch, so wäre es irrational, diesen Wunsch zu haben, ohne der Überzeugung zu sein, daß scine Erfüllung befriedigen wird. Das, oder vielmehr dessen probabilistische Fntsprechung, ist der Inhalt von (3); und dies stimmt mit dem überein, was wir in (IVb) festgestellt haben.

W'as ist aber mit dem in (Vb) und am Ende von (IVa) aufkeimenden Verdacht, daß diese Überzeugung keine normale empirische, sondern eine sich selbst bewahrheitende Übcrzeugung ist? Hier gilt es den genauen Inhalt der Überzeugung zu bcachten. Er kann gegeben sein durch (a): „die Realisierung des gewünschten Sachverhalts $A$ wird befriedigen“ oder durch (b): „die Realisierung des Sachverhalts $A$ wird, sofern er gewünscht wird, befriedigen“. Jeder, der (a) glaubt, wird vermutlich auch (b) glauben; und jeder, $\operatorname{der}(\mathrm{b})$ glaubt und sich des Wunsches nach $A$ bewußt ist, wird sicherlich auch (a) glauben. Und trotzdem besteht zwischen (a) und (b) eine subtile Differcnz. W'enn man der kausalen Überzeugung (a) ist und dabei übcrsieht oder lcugnet, daß der Wunsch nach $A$ für die Befricdigung durch $\mathcal{A}$ kausal relcvant ist, dann kann man zwar das bishcrige Bild aufrecht erhalten, aber nur um den Preis, daß die Überzeugung (a) ihre cigene Wahrheit kausal beeinflußt. Ist man hingegen der kausalcn Überzeugung (b), so muß man zwar in Bezug auf den Nutzen von $A$ das alte Bild aufgeben, hat aber den Vorteil, daß (b) nun eine normale empirische Übcrzeugung darstellt. Nur dic zweite Alternative ist haltbar. Die erste untcrgräbt sich selbst; denn sobald man bemcrkt, daß die eigene Überzcugung ihre Wahrheit bedingt, kann man sie nicht länger unqualifizicrt aufrecht crhalten.

Anderungen der $\ddot{U}$ berqeugung, daß die Verwirklichung eines Wunsches befriedigt: Wenn wir gesagt haben, daß diese Überzeugung den W'unsch notwendig begleitet, so sollte das nicht heißen, daß sie ein Produkt dieses Wunsches 
ist. Im Gegenteil, in der Lesart (b) ist sie cine normale Überzeugung mit normalen Erkenntnisbedingungen. Sie kann durch Erfahrung begründet sein oder nicht; sie kann falsch sein; man kann herausfinden, daß sic falsch ist; und, um es so allgemein zu sagen, daß auch der probabilistische Fall abgedeckt ist, sie kann sich auf der Grundlage neuer Erfahrungen entsprechend den allgemcinen Regeln rationaler Überzeugungsänderung festigen oder verlieren. Welche Folgen hat eine solche Änderung für die Wünsche? Auch hier sind wieder zwei Fälle zu unterscheiden.

Der Normalfall ist, daß eine Überzcugungsänderung nur eine Neuberechnung der erwarteten Nutzenwerte nach sich zieht. Das kann sogar dann der Fall sein, wenn sich eine Annahme über zukünftige Befriedigung verändert. Zum Beispiel bestclle ich in einem Rcstaurant ein exotisches und mir völlig unbckanntes Gericht. Ich probiere es, es schmeckt mir nicht besonders, und so revidiere ich meine Überzeugung über dic Befriedigungswirkung dieses Gerichts und demgcmäß auch scinen erwarteten Nutzen. Dieser Fall läßt sich aber nur deshalb so einfach beschreiben, weil der Zirkel hier nicht auftritt; in der Regel beeinflußt eben der Wunsch nach einem Gericht nicht, wie es schmecken wird.

Ganz anders verhält es sich, wenn der Überlegungszirkcl auftritt. Die Änderung einer Überzeugung über zukünftige Befriedigung, d. h. der in (3) involvierten Wahrscheinlichkeiten, erzeugt dann eine schwierige Lage; der kontrollierende W'unsch nach Befriedigung und die kontrollierten Wünsche nach anderen Dingen sind dann aus dem Gleichgewicht und lassen sich rationalerweise nicht länger aufrechtcrhaltcn. Irgendwie sind sie zu ändern, irgendwo muß ein neues Gleichgewicht gefunden werden; doch ist ganz unklar, wie man sie ändern und wo das Gleichgewicht zu finden sein soll. Jeder kennt die Erfahrung des I.cerlaufs - wenn z. B. die Faszination des Rock'n'roll verblaßt oder cine Liebe verschlissen ist - und den oft quälenden Prozcß einer Neuausrichtung der eigenen Wünsche.

Die Gleichung (3) als Modell der praktischen Überlegung: Dic Gleichungen (1) und (2) liefern Regeln des praktischen Überlegens; sie sagen uns, wie man extrinsische und Gesamtnutzenwerte ableitet. Man könnte daher dazu neigen, die Gleichung (3), die im Zirkelfall die Gleichungen (1) und (2) ersetzt, ebenso aufzufasscn, nämlich als die Regel: Löse (3); gibt es keine Lösung, so ist nichts zu machen (obgleich dies durch geeignete Stetigkcitsannahmen ausgeschlossen werden könnte); gibt cs eine Lösung, so suche die maximale Lösung für $U^{\prime}(\Lambda)$, denn bei ihr ist die erwartete Befriedigung am höchsten! Wäre dies ein durchführbares Überlegungsmodell, so wäre es sinnvoll, die zugehörige mathematischc Thcorie aus- 
zuarbeiten. Aus zwei Gründen erscheint uns dies jedoch äußerst fragwürdig.

Erstens stehen die relevanten Wahrscheinlichkeiten kaum zur Verfügung; sie zu kennen, würde bedeuten, für jeden beliebigen Sachverhalt $A$ auf die folgende Frage eine Antwort zu haben: „Wenn ich $A$ so und so stark wünschte, wie wahrscheinlich ist es dann, daß $A$ mich in dem und dem $\mathrm{MaBe}$ befriedigen würde?" Nun kennen wir aber einfach viele Arten von Befriedigung nicht und können sie nicht einstufen. Wie fühlt man sich als Frau von N. N. oder als chinesischer Reisbauer oder als HimalayaKletterer? Schwer zu sagen. Vollends hoffnungslos wird es, wenn auch noch der Einfluß der Wünsche zu berücksichtigen ist. Wie befriedigend wäre das Klettern im Himalaya, wenn man es gerne täte? Uns schcint, wir können solche Fragen nur beantworten, wenn es um Arten von Befriedigung geht, die wir schon kennen, und um Typen von Wünschen, die wir schon verfolgt haben; alle anderen W'ahrscheinlichkeiten wären pure Raterei.

Zweitens ist die $\Lambda$ usbildung von Zielen und Zwecken, d. h. von nicht abgeleiteten Wünschen, ausgesprochen dunkel. Die Vorstellung, man könnte sich, sozusagen durch schiere Willenskraft, die von einer solchen Überlegung empfohlcnen Wünsche zu eigen machen, scheint doch rcichlich abwegig. Von daher ist es ausgesprochen zweifelhaft, daß eine solche Überlegung den Einfluß auf die Einstellungen und Handlungen des Subjekts haben kann, aus dem sie erst ihren Sinn bezöge.

Rationale und wabre Wünsche: Wenn die Formel (3) nicht als ein Modell der praktischen Überlegung zu verstehen ist, was leistet sie dann? Sie beschreibt ein I.ernmodell, zumindest in dem schwachen Sinn, daß nach ihr Wünsche, die nicht im Gleichgewicht sind, rationalerweisc zu verändern sind und daß diese Veränderung sich auf cin neues Gleichgewicht zuzubewegen hat.

Tatsächlich verfügen wir mit (3) über einen reicheren Begriff rationaler Wünsche. Im Rahmen des bisherigen Bildes gab es keine Möglichkeit, intrinsische W'ünsche oder Nutzenwerte als rational oder irrational zu qualifızieren; nur für extrinsische Wünsche gab es dafür eincn präzisen Sinn. Unser verbesscrtes Bild enthält jedoch zudem für kontrollierte wie für kontrollierende Wünschc einen Rationalitätsstandard (und eröffnet dadurch die Möglichkcit, einen Begriff von rationalen Gefühlen zu bilden). ${ }^{32}$ Anders gesagt: (3) erweitert unsere Möglichkeiten zur Kritik von Wünschen, und zwar zu einer Kritik, die sich nicht auf moralische oder

32 W'ir denken dabei gerade an den in Anmerkung 30 dargelegten Sachverhalt. 
andere externe Standards stützt, sondern sich ausschließlich an die in der Person selbst licgenden Maßstäbe hält. ${ }^{33}$

Insofern die Überzeugung, daß die Erfüllung der eigenen Wünsche befriedigen wird, wahr sein kann und die Wahrscheinlichkeiten in (3) objektiv sein können, verleiht (3) auch der Idee wahrer Wünschc genauere Bedeutung: Unsere wahren Wünsche sind jene, die ausgehend von wahren Überzeugungen in einem maximalen Gleichgewicht sind. Wir sind rationalerweise auf der Suche nach unseren wahren Wünschen; da abcr Befriedigung auf immer wieder andere Weise zustande kommt, muß die Suche immer wieder von neuem anheben. Die Gleichung (3) ist uns dabci nicht wirklich eine praktische Hilfe, aber sie vcrmittelt uns eine gewisse theorctische Einsicht in das, wonach wir suchen.

\section{Literatur}

Bentham, J.: An Introduction to the Principles of Morals and Legislation. $\mathrm{Hg}$. von J. H. Burns and H. L. A. Hart. London 1970.

Burgc, T:: Individualism and the Mental. Midwest Studies in Philosopby IV (1979), S. $73-121$.

Butler, J.: Fiftecn Sermons Preached at the Rolls Chapel. In Butler's Sermons and Dissertation on V'irtue. Hg. von W. R. Matthews. London 1949.

Campbell, R./Sowden, L. (Hg.): Paradoxes of Rationality and Cooperation. Vancouver 1985.

Churchland, P. M.: The Logical Character of Action-Explanations. Philosophical Review 79 (1970) S. 214-236, dt. Übers. in: Analytische llandlungstheorie. Band 2: Handlungserklärungen. Hg. von A. Beckermann. Frankfurt a. M. 1977, S. $304-331$.

Davidson, D.: Essay's on Actions and Events. Oxford 1980, dt. Übers.: Handlung and Ereignis. Frankfurt a. M. 1985.

Eells, E.: Rational Decision and Causality. Cambridge 1982.

Elster, J.: Sour Grapes. Cambridge 1983.

Gosling, J. C. B.: Pleasure and Desire: The Case for Hedonism Reviewed. Oxford 1969. Griffin, J.: Wellbeing. Oxford 1986.

Jeffrey, R. C.: The Jogic of Decision. Chicago 1965. 2. erw. Ausg. 1983, dt. Ubers.: Logik der Entscheidungen. München 1967.

Kant, l.: Kritik der praktischen Vernunft. 1788.

Kusser, A.: Dimensionen der Kritik von Wïnschen. Frankfurt a. M. 1989.

Lewis, D.: Desire as Bclief. Mind 97 (1988), S. 323-332.

Michael, R. T./Becker, G. S.: On the Ncw Theory of Consumer Behavior. Swedisb Journal of Economics 75 (1973), S. 373-396.

Mill, J. St.: Der Utilitarismus. Stuttgart 1976.

33 Dieser Punkt ist in Kusser (1989), S. 185-193, ausführlicher dargclegt. 
Von Neumann, J./Morgenstcrn, O.: Theory of the Games and Economic Behavior. Princcton 1944.

Pears, D.: Motivated Irrationality. Oxford 1986.

Pigou, A. C.: The Fconomics of Welfare. New York 1920.

Raiffa, H.: Decision Analysis. Reading 1969, dt. Übers.: Einfübrung in die Entscheidungstheorie. München 1973.

Rawls, J.: $A$ Theory of Justice. Cambridge, Mass. 1971.

Russell, B.: The Analysis of Mind. London 1921.

Samuelson, P. A.: Foundations of Economic Analysis. Cambridgc 1947.

Savage, L. J.: The Foundations of Statistics. New York 1954, 2. Aufl. 1972.

Sen, A. K.: Bchaviour and the Concept of Prefcrence. Econometrica 40 (1973), S. $241-259$.

Sen, A. K.: Plural Utility. Proceedings of the Arisfotelian Society 81 (1980/81), S. $193-215$.

Sidgwick, H.: The Methods of Ethics. 7. Aufl. London 1962.

Skyrms, B.: Pragmatics and Empiricism. New Haven 1984.

Spohn, W.: Grundlagen der Entscheidungstheorie. Kronberg/Ts. 1978.

Spohn, W.: Stochastic Independence, Causal Independence, and Shieldability. Journal of Philosophical Logic 9 (1980), S. 73-99.

Spohn, W.: Direct and Indirect Causes. Iopoi 9 (1990), S. 125-145.

Tugendthat, E..: Probleme der Ethik. Stuttgart 1984.

Tversky, A./Kahneman, D.: The Framing of Decisions and the Psychology of Chuice. In: Rational Choice. Hg. von J. Elster. New York 1986. 